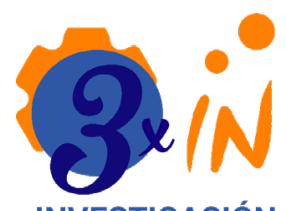

INVESTIGACIÓN e INNOVACION en INGENERIAS ISSN2344-8652

Recibido: 08/07/2021 Aceptado: 09/09/2021 Publicado: 26/11/2021

Correspondencia de autores: njrodriguez@mail.uniatlantico.edu.co

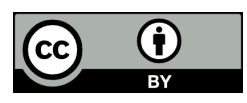

Copyrith 2020

by Investigación e Innovación en Ingenierías

\section{Diseño de un plan de mantenimiento preventivo aplicado a instrumentos no automáticos de pesaje en los laboratorios de la Universidad del Atlántico \\ Design of a preventive maintenance plan applied to non-automatic weighing instruments in the laboratories of the Atlantic Universitya}

\author{
Norberto José Rodríguez-Medina ID Albeiro Javier Fernández-Morenoa \\ Cristian Antonio Pedraza-Yepes \\ Universidad del Atlántico, Colombia \\ José Daniel Hernández-Vásquez \\ Universidad Antonio Nariño, Colombia
}

\begin{abstract}
Objective: Diseñar un plan de mantenimiento preventivo para instrumentos no automáticos de pesaje en laboratorios del programa de nutrición y dietética de la Universidad del Atlántico. Metodología: Se realizó el inventario de 29 instrumentos no automáticos de pesaje con sus respectivas características, con el fin de analizar los errores máximos permitidos y tolerancias según clase (I, II, III y IV); y determinar las actividades de mantenimiento basadas en manuales de diversas marcas de fabricante y conocimientos empíricos para diseñar las respectivas órdenes de trabajo. Resultados: Se seleccionaron 5 instrumentos para aplicar el plan de mantenimiento, incluyendo calibración y aplicación de métodos expuestos por la OIML-D10 de 2017 para determinar las periodicidades de calibración adecuadas. Se realizó el diseño de un Software con información de todas las actividades de mantenimiento para los distintos tipos de equipos ubicados en las instalaciones de los laboratorios y equipos en general, que también es capaz de determinar la periodicidad de calibración basada en el método de Schumacher. Conclusiones: Se determinó que la Universidad requiere con urgencia un plan de mantenimiento para los equipos de sus instalaciones. Se realizó un análisis económico donde se encontró factible el seguimiento de este plan para mantener en óptimas condiciones los equipos y cumplir con las normativas de calidad necesarias para lograr la acreditación de todos sus programas. La presente investigación servirá en un futuro para el diseño de un plan de mantenimiento general para todos los equipos ubicados en las instalaciones de la Universidad del Atlántico.
\end{abstract}

Keywords: Metrología, mantenimiento industrial, métodos estadísticos, diseño de Software.

Resumen

Objetivo: Design a preventive maintenance plan for non-automatic weighing instruments in laboratories of the nutrition and dietetics program of the Universidad del Atlántico. Methodology: An inventory was made of 29 non-automatic weighing instruments with their respective characteristics, in order to analyze the maximum permissible errors and tolerances according to class (I, II, III and IV); and to determine the maintenance activities based on manufacturers manuals and empirical knowledge, in order to design the respective work orders. Results: Five instruments were selected to implement the maintenance plan, including calibration and application of methods set out by the 2017 OIML-D10 to determine appropriate calibration intervals. A software design was made through the $\mathrm{C}++_{+}$ interface with the information of all the maintenance activities for the different types of equipment located in the laboratory facilities and equipment in general, which is also capable of determining the calibration periodicities based on the Schumacher method. Conclusions: It was determined that the University urgently requires a maintenance plan for the equipment in its facilities. An economic analysis was carried out where it was found feasible to follow up this plan to keep the equipment in optimal conditions and comply with the quality standards required to achieve accreditation for all its programs. The present investigation will serve in the future for the design of a general maintenance plan for all the equipment located in the facilities of the Universidad del Atlántico.

Palabras clave: Metrology, industrial maintenance, statistical methods, software design.

Como citar (IEEE): N. Rodríguez-Medina., A. Fernández-Moreno., C. Pedraza-Yepes y J. Hernández-Vásquez “Diseño de un plan de mantenimiento preventivo aplicado a instrumentos no automáticos de pesaje en los laboratorios de la Universidad del Atlántico", Investigación e Innovación en Ingenierías, vol. 9, n², 192-207, 2021. DOI: https://doi.org/10.17081/invinno.9.2.4517 
Diseño de un plan de mantenimiento preventivo aplicado a instrumentos no automáticos de pesaje en los

\section{Introducción}

El mantenimiento industrial es un campo de la ingeniería de gran interés y con amplia repercusión económica. A lo largo de la historia se han desarrollado diferentes metodologías y niveles de aplicación de los sistemas de gestión de mantenimiento industrial. Las industrias actualmente encuentran indispensable la aplicación de un plan de mantenimiento preventivo para el desarrollo de sus actividades, razón por la cual destinan un porcentaje amplio de sus recursos al mantenimiento [1].

Los instrumentos de medición en los laboratorios son herramientas muy precisas para el desarrollo de investigaciones relacionadas a industrias tales como: las farmacéuticas y alimenticias; y de ciencias como: la física y química; por lo que estos instrumentos no pueden tener un margen de error significativo y se le deban realizar mantenimientos preventivos y correctivos [2], ya que si se ignorasen, las mediciones se transformarían en un proceso de especulaciones que con el tiempo irían perdiendo su exactitud y veracidad [3].

Como la Universidad del Atlántico se encuentra actualmente en búsqueda de certificar todos los programas que esta universidad ofrece, y que hasta la fecha de presentado este artículo 22 de los 32 programas existentes ya están acreditados [4], se propuso realizar un plan de mantenimiento preventivo a instrumentos de pesaje de masa de los laboratorios de la alma mater que ayudaría a que algunos requisitos de acreditación de normas como la ISO 9001 [5] sean cumplidos, además de ayudar a alcanzar una mayor calidad de educación e investigación.

El objetivo principal de esta investigación es desarrollar para los laboratorios de la Universidad del Atlántico un plan de mantenimiento preventivo aplicado a los instrumentos no automáticos de pesaje trazando como objetivo secundario una metodología que permita reevaluar y establecer los intervalos de tiempos en la calibración de instrumentos tomando como soporte principal los métodos de control metrológico propuestos por la OIML (organización internacional de metrología legal) en la guía D10 [6].

Se han desarrollado investigaciones previas que soportan la implementación de este proyecto, como lo son la realizada por D. Medrano y B. Vega [7] en 2012, donde desarrollaron un sistema de mejoramiento continuo de las actividades de mantenimiento con planeación y ejecución del mantenimiento para los equipos del laboratorio de procesos de manufactura en la Escuela Politécnica del Ejército, basándose en actividades principales como caracterización de equipos, verificación metrológica, elaboración de documentos y formatos, y un análisis económico.

Otro de los objetivos planteados en este proyecto es el desarrollo de una interfaz que contenga la información de todas las actividades de mantenimiento y que cuente con la opción de determinar una periodicidad de calibración adecuada basado en métodos estadísticos y en historiales de calibración de los equipos.

En 2014, E. Dumaguala [8] desarrolló un plan de mantenimiento para los laboratorios de ingeniería en la Universidad Politécnica Salesiana. La gestión del mantenimiento que se ha aplicado en este proyecto tiene tres etapas: La primera es el levantamiento del inventario de máquinas y equipos con sus respectivas características técnicas; la segunda etapa, donde se realizó una planificación del mantenimiento preventivo para cada máquina, donde se requirió conocer los objetivos de cada laboratorio y determinar un objetivo de mantenimiento para categorizar las máquinas. Con la categorización de las máquinas se asignaron las diferentes tareas preventivas con sus instrucciones, lo cual permitió definir los insumos y herramientas necesarias para la ejecución del mantenimiento; y por último, la tercera etapa del proyecto, donde se 
realizó la implementación de la gestión mediante el software de mantenimiento sisMAC. En esta etapa se ingresó al software la información de los laboratorios aplicando las condiciones previamente definidas. El resultado de la programación del mantenimiento de manera automática permite la gestión de insumos, herramientas para cada semestre.

En 2018, N. Montealegre [9] diseñó un plan de mantenimiento para los equipos del laboratorio de ingeniería mecánica de la Universidad Tecnológica de Pereira. La investigación, muestra la descripción de las actividades desarrolladas durante los cinco meses de la práctica empresarial. Además, con el propósito de dar cumplimiento a los objetivos específicos planteados se establecieron cinco actividades principales: Como primera actividad, la recolección de información registrada y almacenada, adquirida en el transcurso de la vida útil de cada equipo en cada uno de los laboratorios, generando así un listado de equipos que conforman el plan de mantenimiento preventivo. Como segunda actividad, diligenciar la ficha técnica y ordenar la información de registros de mantenimientos en las hojas de vida del listado de equipos. En la tercera actividad se genera un plan de mantenimiento preventivo teniendo en cuenta las tareas básicas de mantenimiento, las cuales son necesarias realizar con una frecuencia estipulada en cada equipo. Como cuarta actividad, se implementa una prueba piloto en uno de los laboratorios de la Facultad mediante el software SMplus pro 3.0, obteniendo así una observación del estado actual en el que se encuentra la gestión de mantenimiento preventivo en los laboratorios. Finalmente, se establecen los equipos necesarios a reposición teniendo en cuenta los diferentes criterios de acuerdo al contexto en que se desarrolla la práctica empresarial.

Investigaciones, como la realizada por E. Cruz y V. Branda [10], avalan la importancia del uso de la guía OIML D10. Ellos establecieron bajo esta guía, los intervalos de calibración de instrumentos de medición de presión estática y temperatura en una comercializadora de gas natural en Brasil, siguiendo y evaluando cuatro métodos distintos de esta guía: Shumacher, Drift, Weighted y Poisson; siendo este último el escogido para seleccionar el intervalo que fue de 322 días.

En 2017 Jing Wang, Qi Zhang y Wei Jiang [11], notaron que si el intervalo de calibración es demasiado largo, el riesgo de exceder la tolerancia incrementará; y por el contrario, si el intervalo es demasiado corto, habrá un desperdicio de los recursos y el uso normal del instrumento se verán afectados. En su investigación que buscaba la optimización de los intervalos de calibración para equipos de prueba automáticos, obtuvieron que los intervalos de calibración para un analizador de señales radio frecuencia es de 14 meses; para un osciloscopio es 15 meses; para un multímetro digital 17 meses, para un generador de ondas arbitrarias 12 meses; para un módulo A/D 7 meses y para un módulo D/A 8 meses.

\section{Metodología}

\section{Inventario de equipos}

Los laboratorios del programa de nutrición y dietética de la Universidad del Atlántico actualmente cuentan con 8 tipos diferentes de balanzas dentro de sus instalaciones, las cuales son:

- Balanzas digitales.

- Balanzas grameras análogas.

- Balanzas grameras digitales.

- Balanzas de peso corporal. 
- Balanzas pesa bebés.

- Balanzas médicas.

- Balanzas verticales.

- Balanzas granatarias.

En la Tabla 1 se especifica el inventario total de instrumentos no automáticos de pesaje de las instalaciones de los laboratorios del programa de nutrición y dietética de la Universidad del Atlántico.

Tabla 1. Inventario instrumentos no automático de pesaje de laboratorios de nutrición y dietética de Universidad del Atlántico.

\begin{tabular}{|c|c|c|c|c|c|c|c|c|}
\hline İtem & Descripción & Marca & Modelo & Cantidad & Estado & Capacidad & Resolución & Ubicación \\
\hline 1 & $\begin{array}{c}\text { Balanza peso } \\
\text { corporal }\end{array}$ & No presenta & $\begin{array}{c}3865 \text { HOM } \\
1697\end{array}$ & 1 & Bueno & $180 \mathrm{~kg}$ & $100 \mathrm{~g}$ & Consultorio \\
\hline 2 & $\begin{array}{c}\text { Balanza pesa } \\
\text { bebe }\end{array}$ & $\begin{array}{l}\text { Health o } \\
\text { meter }\end{array}$ & & 1 & Bueno & $25 \mathrm{~kg}$ & $125 \mathrm{~g}$ & Consultorio \\
\hline 3 & $\begin{array}{l}\text { Balanza } \\
\text { vertical }\end{array}$ & $\begin{array}{c}\text { Health o } \\
\text { meter }\end{array}$ & & 1 & Regular & $140 \mathrm{~kg}$ & $100 \mathrm{~g}$ & Consultorio \\
\hline 4 & $\begin{array}{l}\text { Balanza } \\
\text { medica }\end{array}$ & Detecto & & 3 & Bueno & $110 \mathrm{~kg}$ & $100 \mathrm{~g}$ & Lab 101 \\
\hline 5 & $\begin{array}{c}\text { Balanza } \\
\text { medica }\end{array}$ & Detecto & & 4 & Regular & $220 \mathrm{~kg}$ & $100 \mathrm{~g}$ & Lab 101 \\
\hline 6 & $\begin{array}{c}\text { Balanza pesa } \\
\text { bebe }\end{array}$ & $\begin{array}{l}\text { Health o } \\
\text { meter }\end{array}$ & $\begin{array}{c}3865 \text { HOM } \\
1697\end{array}$ & 3 & Bueno & $25 \mathrm{~kg}$ & $100 \mathrm{~g}$ & Lab 101 \\
\hline 7 & Balanza digital & Bernalo & ACS-30/15 & 1 & Bueno & $30 / 15 \mathrm{~kg}$ & $200 / 100 \mathrm{~g}$ & Lab 104 \\
\hline 8 & Balanza digital & Bernalo & JCS-A & 1 & Bueno & $30 \mathrm{~kg}$ & $1 \mathrm{~g}$ & Lab 104 \\
\hline 9 & $\begin{array}{c}\text { Balanza } \\
\text { granataria }\end{array}$ & No presenta & & 1 & Bueno & $2,61 \mathrm{~kg}$ & $0.1 \mathrm{~g}$ & Lab 104 \\
\hline 10 & $\begin{array}{c}\text { Gramera } \\
\text { manual }\end{array}$ & $\begin{array}{l}\text { Use for } \\
\text { family }\end{array}$ & & 10 & Bueno & $5 \mathrm{~kg}$ & $40 \mathrm{~g}$ & Lab 104 \\
\hline 11 & $\begin{array}{c}\text { Gramera } \\
\text { manual }\end{array}$ & No presenta & & 1 & Bueno & $10 \mathrm{~kg}$ & $50 \mathrm{~g}$ & Lab 104 \\
\hline 12 & $\begin{array}{l}\text { Gramera } \\
\text { digital }\end{array}$ & No presenta & & 2 & Bueno & $10 \mathrm{~kg}$ & $1 g$ & Lab 104 \\
\hline
\end{tabular}

Fuente: Elaboración propia

\section{Clasificación de balanzas Determinación de actividades de mantenimiento}

Ya teniendo el inventario total de los instrumentos que se utilizarán para realizar el plan de mantenimiento con sus respectivas características, se siguen ciertas estrategias y técnicas para determinar las actividades que se llevarán a cabo.

Para escoger las técnicas que mejor se adapten a un plan de mantenimiento y cumpla con los requisitos de gestión metrológica para la calibración de instrumentos de pesaje no automáticos [12], primero hay que saber cuáles son: 
- Técnica basada en instrucciones de fabricantes: La cuál es la más sencilla de todas, ya que nos basaremos en las recomendaciones por los fabricantes. Consiste en determinar y elaborar las tareas que debemos aplicar en el plan de mantenimiento para los equipos, basándonos en las manuales de fabricante.

- Técnica basada en protocolos genéricos por tipo de equipo: Consiste en que cuando se escoge un equipo, podemos basarnos en actividades generales específicas para equipos del mismo estilo.

- Técnica basada en RCM: Es la más detallada y exhaustiva de las tres técnicas, donde para llevar el plan de mantenimiento a cabo debemos realizar lo siguiente:

$\gg$ Una lista con todos los sistemas y sub sistemas con los que cuenta la empresa, así como también una lista con los principales equipos que conforman cada sub sistema.

$\gg$ Determinar y estudiar las especificaciones técnicas de los equipos de cada sistema y sub sistema.

$\#$ Definir todas las funciones primarias y secundarias que se pueden sacar de la información de las especificaciones anteriormente nombradas.

$\gg$ Determinar los modos de fallo de los equipos, que no son más que aquellos que impedirían que se logre la función de estos.

$\gg$ Realizar un listado con medidas preventivas para evitar estos modos de fallo.

$\gg$ Agrupar todo el conjunto de tareas de mantenimiento por tipos, donde podemos incluso realizar un documento con el plan de formación y listado de herramientas y recursos que necesitamos para llevar todo el mantenimiento a cabo.

En este proyecto, se describirá una serie de actividades que se deben seguir para realizar los mantenimientos preventivos respectivos para cada tipo de balanza existente en el inventario de instrumentos de los laboratorios basándonos en las técnicas basadas en manuales de fabricante y en protocolos genéricos debido a su nivel de complejidad no tan alto [13].

\section{Métodos de cálculo de periodicidad de calibración}

Para determinar la periodicidad de calibración se tomará como base los criterios de los métodos descritos en la guía OIML-D10 de Carta de control y Schumacher.

El método de Carta de control [14] se basa en los valores de incertidumbre y tolerancias arrojados en los certificados de calibración y que son graficados dependientes al tiempo. Este tipo de gráfico permite observar si el equipo presenta tendencias o patrones de comportamiento a lo largo del tiempo, utilizado una Figura de desarrollo de los errores e incertidumbres con respecto a la fecha de calibración.

El método de Schumacher [15] permite establecer un nuevo periodo de calibración por medio de la interpretación de la siguiente Tabla: 
Tabla 2. Explicación de método Schumacher

\begin{tabular}{|c|c|c|c|}
\hline \multirow{2}{*}{ Ciclos anteriores } & \multicolumn{2}{|c|}{ Condiciones del instrumento } \\
\cline { 2 - 4 } & $\mathrm{A}$ & $\mathrm{F}$ & $\mathrm{C}$ \\
\hline $\mathrm{CCC}$ & $\mathrm{P}$ & $\mathrm{D}$ & $\mathrm{E}$ \\
\hline $\mathrm{FCC}$ & $\mathrm{P}$ & $\mathrm{D}$ & $\mathrm{E}$ \\
\hline $\mathrm{ACC}$ & $\mathrm{P}$ & $\mathrm{D}$ & $\mathrm{P}$ \\
\hline $\mathrm{CF}$ & $\mathrm{M}$ & $\mathrm{M}$ & $\mathrm{P}$ \\
\hline $\mathrm{CA}$ & $\mathrm{M}$ & $\mathrm{M}$ & $\mathrm{P}$ \\
\hline $\mathrm{FC}$ & $\mathrm{P}$ & $\mathrm{M}$ & $\mathrm{P}$ \\
\hline $\mathrm{FF}$ & $\mathrm{M}$ & $\mathrm{M}$ & $\mathrm{P}$ \\
\hline $\mathrm{FA}$ & $\mathrm{M}$ & $\mathrm{M}$ & $\mathrm{P}$ \\
\hline $\mathrm{AC}$ & $\mathrm{P}$ & $\mathrm{D}$ & $\mathrm{P}$ \\
\hline AF & $\mathrm{M}$ & $\mathrm{M}$ & $\mathrm{P}$ \\
\hline AA & $\mathrm{M}$ & $\mathrm{M}$ & \\
\hline
\end{tabular}

Fuente: Adaptado de [15]

En este enfoque los instrumentos se clasifican de acuerdo con las condiciones en que se encuentran con base en los registros históricos de calibración en:

- A: Presenta daño o defecto.

- C: Está conforme a los criterios de aceptación.

- F: No atiende los criterios de aceptación.

Con al menos tres certificados de calibración sucesivos es posible tomar las siguientes decisiones basado en la Tabla anterior.

- E: Aumentar la periodicidad en un $20 \%$.

- D: Disminuir la periodicidad en un $10 \%$.

- M: Máxima reducción de la periodicidad en $35 \%$.

- P: Permanecer en el mismo lapso de calibración.

\section{Diseño de software}

Con las actividades ya determinadas se procedió a diseñar un software a través de la interfaz de C++ que contiene una base de datos con todas las respectivas tareas de mantenimiento para cada tipo de balanza existente en los laboratorios de la Universidad del Atlántico, así como también actividades generales para cualquier otro tipo de balanza distinta perteneciente a las instalaciones del alma mater.

Este software viene además con la opción de determinar la periodicidad de calibración de instrumentos basándose en el método de Schumacher, para lo cual se necesita que el instrumento para que el que se requiera calcular la nueva periodicidad de calibración cuente con los últimos tres certificados de calibración. Cabe resaltar que esta opción sirve para determinar no solo la periodicidad de calibración de instrumentos no automáticos de pesaje sino también para determinar la periodicidad de calibración de otros tipos de equipos. 
La razón principal por la cual se decidió llevar a cabo el diseño de esta base de datos por medio del programa $\mathrm{C}_{+}+$es que este es un ejecutable práctico y fácil de manejar que no necesita ser instalado y tampoco tener instalado el mismo programa de $\mathrm{C}++^{+}$lo cual lo vuelve una gran ventaja con respecto a otros programadores como lo son MatLab, JavaScript o SIMULINK.

Figura 1. Menú Software base de datos de mantenimiento para balanzas de la Universidad del Atlántico.

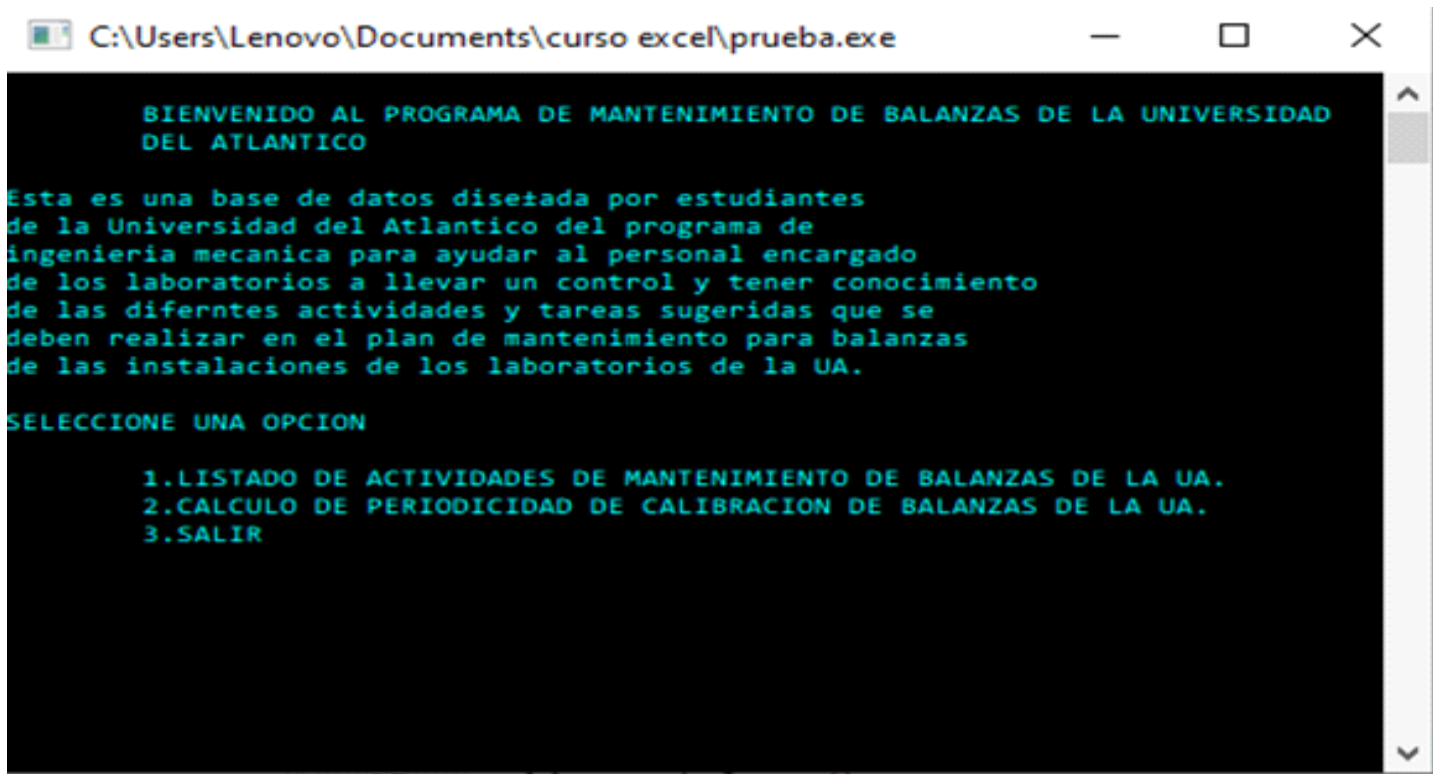

Fuente: Elaboración propia

El programa cuenta con los siguientes menús:

1) Plan de mantenimiento para balanzas.

Dentro de este menú se encontrarán las distintas actividades para los distintos 8 tipos de balanzas existentes en los laboratorios de Nutrición y Dietética de la Universidad del Atlántico y para balanzas en general. Dentro de cada uno de los menús de balanzas se pueden averiguar las actividades de inspección, limpieza, ajuste y verificación y la periodicidad de calibración de cada tipo de balanza.

2) Cálculo de periodicidad de calibración para balanzas.

En este menú se podrá hacer consulta de la periodicidad de calibración de instrumentos de pesaje existentes en los laboratorios de Nutrición y Dietética de la Universidad del Atlántico, así como también de balanzas que lleguen nuevas a las instalaciones. Estos menús para determinar la periodicidad de calibración para balanzas nuevas cuentan con algoritmos que necesitan información de ciertas variables para determinar una cantidad de días. 
Diseño de un plan de mantenimiento preventivo aplicado a instrumentos no automáticos de pesaje en los

\section{Resultados}

\section{Desarrollo de actividades de mantenimiento.}

En la Tabla 3 se describen las actividades sugeridas para el plan de mantenimiento para instrumentos no automáticos de pesaje ubicados en los laboratorios del programa de nutrición y dietética de la Universidad del Atlántico.

Dichas actividades de mantenimiento así como periodicidad de calibración se basaron en estrategias expuestas en manuales de fabricantes de distintas marcas $[16,17,18,19,20,21,22]$ y conocimiento empírico de expertos en el mantenimiento, reparación y distribución de instrumentos no automáticos de pesaje $[23,24]$.

Tabla 3. Listado de actividades del plan de mantenimiento para equipos no automáticos de pesaje de laboratorios de Universidad del Atlántico

\begin{tabular}{|c|c|}
\hline ACTIVIDAD & DESCRIPCIÓN \\
\hline \multicolumn{2}{|l|}{ INSPECCIÓN } \\
\hline 1 & Inspeccionar las condiciones ambientales en las que se encuentra el equipo. \\
\hline 2 & $\begin{array}{l}\text { Inspeccionar la plataforma o el lugar en el que se encuentre el equipo, siendo esta un lugar nivelado } \\
\text { de alta inercia y que esté libre de equipos u objetos que ejerzan vibración sobre la balanza. }\end{array}$ \\
\hline 3 & Desconectar cable de corriente de la balanza en caso tal se encuentre enchufado. \\
\hline 4 & $\begin{array}{l}\text { Verificar que el equipo cuente con todos los componentes completos, y que la placa de identificación } \\
\text { se encuentre adherida al mismo. }\end{array}$ \\
\hline 5 & Inspeccionar el sistema mecánico, eléctrico o electrónico según corresponda. \\
\hline 6 & $\begin{array}{l}\text { Levantar platillo de pesaje e inspeccionar el estado del brazo donde descansa dicho plato en busca } \\
\text { de algún tipo de defecto superficial que pueda afectar el buen funcionamiento de la balanza. }\end{array}$ \\
\hline 7 & $\begin{array}{l}\text { Realizar un examen minucioso de forma visual de cada componente del equipo en búsqueda de } \\
\text { rasguños, golpes, hendiduras, decoloración, oxidación o cualquier otro factor que afecte la apariencia } \\
\text { e integridad del equipo. }\end{array}$ \\
\hline 8 & Inspeccionar estructura general del equipo, así como también bases y apoyos. \\
\hline 9 & Inspeccionar estado de cojinetes y barras de medición. \\
\hline 10 & Inspeccionar tallimetro y cinta métrica. \\
\hline 11 & $\begin{array}{l}\text { Inspeccionar la aguja y el tablero del medidor de masa en forma de reloj, que se encuentren en } \\
\text { estado de uso, que se logre identificar la resolución que maneja el instrumento. }\end{array}$ \\
\hline 12 & Inspeccionar estado de baterías y porta baterías \\
\hline \multicolumn{2}{|l|}{ LIMPIEZA } \\
\hline 13 & $\begin{array}{l}\text { Se deben realizar todo tipo de limpiezas en el mismo sitio de trabajo de la balanza, sin moverse, } \\
\text { inclinarse o cambiar de lugar ya que una manipulación incorrecta de esta podría significar daños } \\
\text { costosos. }\end{array}$ \\
\hline 14 & $\begin{array}{l}\text { Verificar que el equipo no se encuentre conectado y proteger con plástico todo elemento eléctrico } \\
\text { y/o electrónico. }\end{array}$ \\
\hline 15 & $\begin{array}{l}\text { Realizar limpieza externa del equipo, quitando el polvo y suciedad con un paño o pedazo de tela } \\
\text { limpio. Nunca se debe soplar para limpiar, ya que la suciedad o los materiales derramados de las } \\
\text { muestras podrían depositarse en el interior de la balanza generando daños mayores. }\end{array}$ \\
\hline
\end{tabular}




\begin{tabular}{|c|c|}
\hline 16 & $\begin{array}{l}\text { Retirar sustancias pegajosas de la superficie, utilizar un paño húmedo sin pelusas y disolvente suave } \\
\text { (isopropanol o etanol al 70\%). No utilizar materiales abrasivos ya que desgastan la resistencia de la } \\
\text { superficie, genera anormalidades en la estructura y afecta la apariencia del equipo. }\end{array}$ \\
\hline 17 & $\begin{array}{l}\text { Desmontar piezas que se puedan quitar sin herramientas y que cuya remoción se describa en el } \\
\qquad \text { manual de fabricante, y realizar limpieza de estas. }\end{array}$ \\
\hline \multicolumn{2}{|l|}{$\begin{array}{c}\text { AJUSTE Y } \\
\text { VERIFICACIÓN }\end{array}$} \\
\hline 18 & $\begin{array}{l}\text { Verificar que la balanza se encuentre nivelada. En balanzas mecánicas, esto se logra mediante } \\
\text { mecanismos de ajuste roscado, ubicados en la base de la balanza. El nivel se logra centrando una } \\
\text { burbuja sobre una escala visible en la parte frontal de la base de la balanza. }\end{array}$ \\
\hline 19 & $\begin{array}{l}\text { Volver a colocar la bandeja de pesaje y encender la balanza y diagnosticar la funcionabilidad de cada } \\
\text { botón con la que cuenta el equipo. }\end{array}$ \\
\hline 20 & $\begin{array}{l}\text { Colocar el interruptor en ON y esperar que se estabilicen los ceros en las pantallas, caso contrario } \\
\text { hacer reset con el botón de TARA. }\end{array}$ \\
\hline 21 & $\begin{array}{l}\text { Verificar la graduación de cero, colocando en cero los controles de la balanza y liberándolo de } \\
\text { todo peso encima. En caso de no estar en cero, para balanzas mecánicas es necesario ajustar el } \\
\text { mecanismo de ajuste de cero que es un tornillo estriado ubicado en posición horizontal cerca al } \\
\text { fulcro. Para esto es necesario bloquear la balanza y ajustar suavemente el citado mecanismo. El } \\
\text { proceso continúa hasta que el cero ajuste correctamente en la escala de lectura. Para balanzas } \\
\text { eléctricas se consigue esto presionando el botón "tara". }\end{array}$ \\
\hline 22 & $\begin{array}{l}\text { Verificar el ajuste de sensibilidad de la balanza colocando un peso patrón conocido equivalente al } \\
\qquad \text { rango de la escala óptica en el platillo. }\end{array}$ \\
\hline 23 & $\begin{array}{l}\text { Colocar pesos conocidos que estén en el rango de pesaje sobre la balanza y diagnosticar su } \\
\text { funcionamiento. Determinar los errores que llegue a presentar. }\end{array}$ \\
\hline 24 & $\begin{array}{l}\text { Verificar el funcionamiento de la balanza, determinar su error de medida realizando múltiples } \\
\text { repeticiones de pesaje, y determinar si requiere de ajuste o corrección. }\end{array}$ \\
\hline 25 & $\begin{array}{l}\text { Colocar la graduación de la década de peso inferior en (1), liberar la balanza y ajustar el punto cero. } \\
\text { Se debe colocar la graduación de la década de peso inferior nuevamente en (0). La balanza debe } \\
\text { marcar 100. En caso marque distinto de } 100 \text { se debe ajustar el control de sensibilidad. Se debe } \\
\text { bloquear la balanza, levantar la cubierta superior y girar el tornillo de sensibilidad. Si la escala } \\
\text { marca más de 100, se debe girar el tornillo en el sentido de las agujas del reloj y si la escala marca } \\
\text { menos de 100, es necesario desenroscar el tornillo. Luego de esto, se debe repetir el proceso hasta } \\
\text { que quede ajustada la balanza. }\end{array}$ \\
\hline 26 & Para balanzas con recipientes, verificar que se encuentren correctamente cerrados. \\
\hline 27 & $\begin{array}{l}\text { Cada vez que se cambie de lugar la balanza, se deben realizar los respectivos ajustes. Estos } \\
\text { ajustes también se deben realizar si las condiciones de trabajo y de ambiente varían afectando el } \\
\text { funcionamiento del equipo. }\end{array}$ \\
\hline 28 & Limpiar la bandeja de pesaje luego de realizada la prueba de peso. \\
\hline 29 & Apagar la balanza, y guardarse en un lugar libre de humedad, riesgos de golpes y radiación de calor. \\
\hline 30 & $\begin{array}{l}\text { Cualquier sustancia que interfiera con los mecanismos de la balanza retarda su respuesta o alteran } \\
\text { definitivamente la medida. En caso se deba realizar, se lubrican los componentes mecánicos internos } \\
\text { que el fabricante indique. }\end{array}$ \\
\hline CALIBRACIÓN & Se debe realizar proceso de calibración con la periodicidad que se sugiere en este documento. \\
\hline
\end{tabular}

\section{Fuente: Elaboración propia}


En la Tabla 5 se describe la periodicidad sugerida para cada una de las actividades mencionadas anteriormente para los distintos tipos de instrumentos de pesaje no automáticos existentes en los laboratorios del programa de Nutrición y Dietética de la Universidad del Atlántico. Para ello, en la Tabla 4 se describen los tipos de balanzas existentes:

Tabla 4. Tipo de balanzas existentes en laboratorios de nutrición y dietética de la Universidad del Atlántico

\begin{tabular}{|c|c|}
\hline BALANZA \# & TIPO DE BALANZA \\
\hline Balanza 1 & Digital. \\
\hline Balanza 2 & Gramera análoga. \\
\hline Balanza 3 & Gramera digital. \\
\hline Balanza 4 & Balanza pesa personas. \\
\hline Balanza 5 & Balanza pesa bebés. \\
\hline Balanza 6 & Balanza médica. \\
\hline Balanza 7 & Balanza vertical. \\
\hline Balanza 8 & Balanza granataria. \\
\hline
\end{tabular}

Fuente: Elaboración propia

Tabla 5. Asignación y periodicidad de actividades de plan de mantenimiento

\begin{tabular}{|c|c|c|c|c|c|c|c|c|}
\hline \multirow[b]{2}{*}{ ACTIVIDAD } & \multicolumn{8}{|c|}{ PERIODICIDAD DE ACTIVIDAD DE MANTENIMIENTO (DIAAS) } \\
\hline & Balanza 1 & Balanza 2 & Balanza 3 & Balanza 4 & Balanza 5 & Balanza 6 & Balanza 7 & Balanza 8 \\
\hline \multicolumn{9}{|l|}{ INSPECCIÓN } \\
\hline 1 & 1 & 1 & 1 & 1 & 1 & 1 & 1 & 1 \\
\hline 2 & 1 & 1 & 1 & 1 & 1 & 1 & 1 & 1 \\
\hline 3 & 1 & $\mathrm{~N} / \mathrm{A}$ & $\mathrm{N} / \mathrm{A}$ & $\mathrm{N} / \mathrm{A}$ & N/A & N/A & $N / A$ & N/A \\
\hline 4 & 1 & 1 & 1 & 1 & 1 & 1 & 1 & 1 \\
\hline 5 & 1 & 1 & 1 & 1 & 1 & 1 & 1 & 1 \\
\hline 6 & 7 & 7 & 7 & 7 & 7 & 7 & 7 & 7 \\
\hline 7 & 1 & 1 & 1 & 1 & 1 & 1 & 1 & 1 \\
\hline 8 & 1 & 1 & 1 & 1 & 1 & 1 & 1 & 1 \\
\hline 9 & $\mathrm{~N} / \mathrm{A}$ & N/A & $\mathrm{N} / \mathrm{A}$ & N/A & $\mathrm{N} / \mathrm{A}$ & 1 & 1 & 1 \\
\hline 10 & $\mathrm{~N} / \mathrm{A}$ & N/A & $\mathrm{N} / \mathrm{A}$ & N/A & 1 & 1 & 1 & $\mathrm{~N} / \mathrm{A}$ \\
\hline 11 & $\mathrm{~N} / \mathrm{A}$ & 1 & 1 & $\mathrm{~N} / \mathrm{A}$ & $\mathrm{N} / \mathrm{A}$ & N/A & $N / A$ & N/A \\
\hline 12 & 7 & N/A & 7 & 7 & $\mathrm{~N} / \mathrm{A}$ & N/A & $\mathrm{N} / \mathrm{A}$ & $\mathrm{N} / \mathrm{A}$ \\
\hline \multicolumn{9}{|l|}{ LIMPIEZA } \\
\hline 13 & 7 & 7 & 7 & 7 & 7 & 7 & 7 & 7 \\
\hline 14 & 7 & $\mathrm{~N} / \mathrm{A}$ & 7 & 7 & N/A & N/A & $\mathrm{N} / \mathrm{A}$ & N/A \\
\hline 15 & 7 & 7 & 7 & 7 & 7 & 7 & 7 & 7 \\
\hline 16 & 7 & 7 & 7 & 7 & 7 & 7 & 7 & 7 \\
\hline 17 & 7 & 7 & 7 & $\mathrm{~N} / \mathrm{A}$ & 7 & 7 & 7 & 7 \\
\hline \multicolumn{9}{|l|}{ AJUSTE } \\
\hline 18 & 7 & 7 & 7 & 7 & 7 & 7 & 7 & 7 \\
\hline
\end{tabular}




\begin{tabular}{|c|c|c|c|c|c|c|c|c|}
\hline 19 & 7 & 7 & 7 & 7 & 7 & 7 & 7 & 7 \\
\hline 20 & 7 & N/A & 7 & N/A & N/A & N/A & N/A & N/A \\
\hline 21 & 7 & 7 & 7 & 7 & 7 & 7 & 7 & 7 \\
\hline 22 & 7 & 7 & 7 & 7 & 7 & 7 & 7 & 7 \\
\hline 23 & 7 & 7 & 7 & 7 & 7 & 7 & 7 & 7 \\
\hline 24 & 7 & 7 & 7 & 7 & 7 & 7 & 7 & 7 \\
\hline 25 & 7 & 7 & 7 & 7 & 7 & 7 & 7 & 7 \\
\hline 26 & N/A & 7 & N/A & N/A & N/A & N/A & N/A & N/A \\
\hline 27 & 7 & 7 & 7 & 7 & 7 & 7 & 7 & 7 \\
\hline 28 & 7 & 7 & 7 & 7 & 7 & 7 & 7 & 7 \\
\hline 29 & 7 & N/A & 7 & 7 & N/A & N/A & N/A & N/A \\
\hline 30 & N/A & N/A & N/A & N/A & N/A & 7 & 7 & 7 \\
\hline CALIBRACIÓN & 340 & 420 & 310 & 420 & 360 & 330 & 320 & 330 \\
\hline
\end{tabular}

\section{Fuente: Elaboración propia}

Una vez asignadas las tareas de mantenimiento correspondiente a cada uno de los distintos tipos de balanzas que se encuentran en los laboratorios, se diseñaron las plantillas correspondientes a las órdenes de trabajo de cada equipo y se aplicó este a todos los equipos, pero donde a solo cinco de estos se les realizó el proceso de calibración por motivos de presupuestos y estado actual de cada equipo.

\section{Aplicación de métodos para el cálculo de periodicidad de calibración.}

Para seleccionar los instrumentos que sirvieron como objeto de estudio para la aplicación de estos métodos se tuvo en cuenta lo siguiente $[15,25,26]$ :

- La incertidumbre de medición requerida por el proceso de calibración.

- El riesgo de que el error total exceda la tolerancia establecida para el proceso de medición y afecte de forma adversa el mismo.

- Los costos financieros para el caso de que las mediciones afecten de forma adversa los resultados.

- El tipo de instrumentos.

- Recomendaciones del fabricante.

- Condiciones de uso.

- Frecuencia de uso.

- Controles de verificación.

- Transporte y conocimiento técnico de los operadores.

- Impacto de los resultados finales.

- Conocimiento e informaciones previas sobre el comportamiento del equipo.

- Recomendaciones del laboratorio metrológico.

- Un mínimo de tres históricos de calibraciones y ajustes realizados.

De acuerdo a los ítems anteriormente mencionados se escogieron los siguientes tres de los cinco equipos calibrados, ya que estos cumplían con un total de mínimo tres históricos de calibraciones: 
- Balanza 1: Balanza médica con capacidad de $140 \mathrm{~kg}$.

- Balanza 2: Balanza pesa bebés con capacidad de 22,68kg.

- Balanza 3: Balanza vertical con capacidad de $200 \mathrm{~kg}$.

\section{Aplicación de método carta de control.}

Se realizó una Tabla de cálculo en Excel donde se ingresan los valores de capacidad, error e incertidumbre obtenidos de los certificados de calibración realizados en los equipos previamente para obtener las Figuras del método de carta de control y se obtuvo lo siguiente:

Figura 1. Comportamiento de calibración de balanza 1

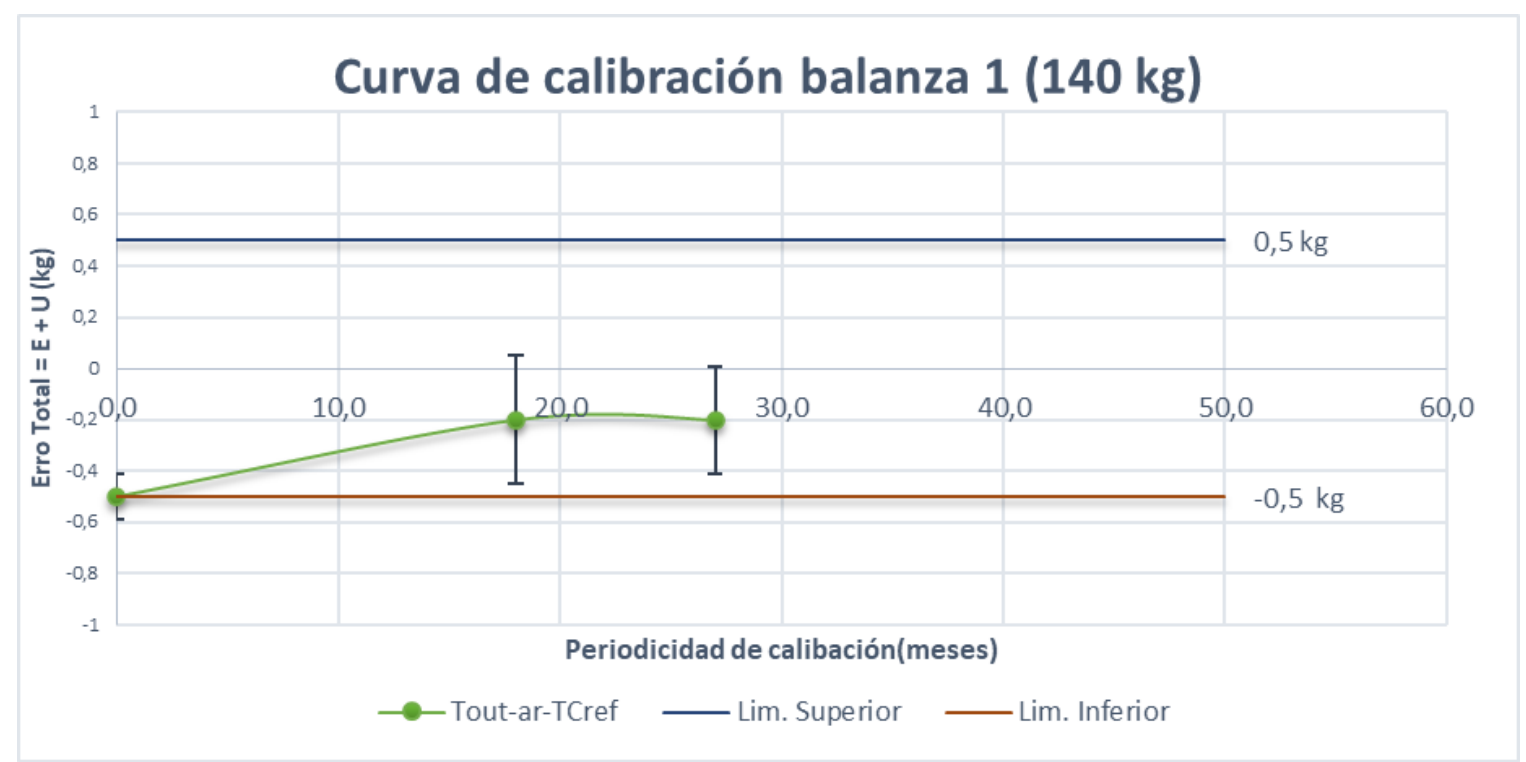

Fuente: Elaboración propia

De la Figura 1, podemos observar que al reducirse su anterior periodicidad de calibración, esta empezó a comportarse de tal manera que los errores arrojados se disminuyeron, por lo que es ideal mantener esta periodicidad y seguir realizándole mantenimiento en ese lapso de tiempo para no afectar su funcionamiento y predecir que se siga observando que los errores se encuentran dentro de los límites de tolerancia del instrumento. 
Figura 2. Comportamiento de calibración de balanza 2

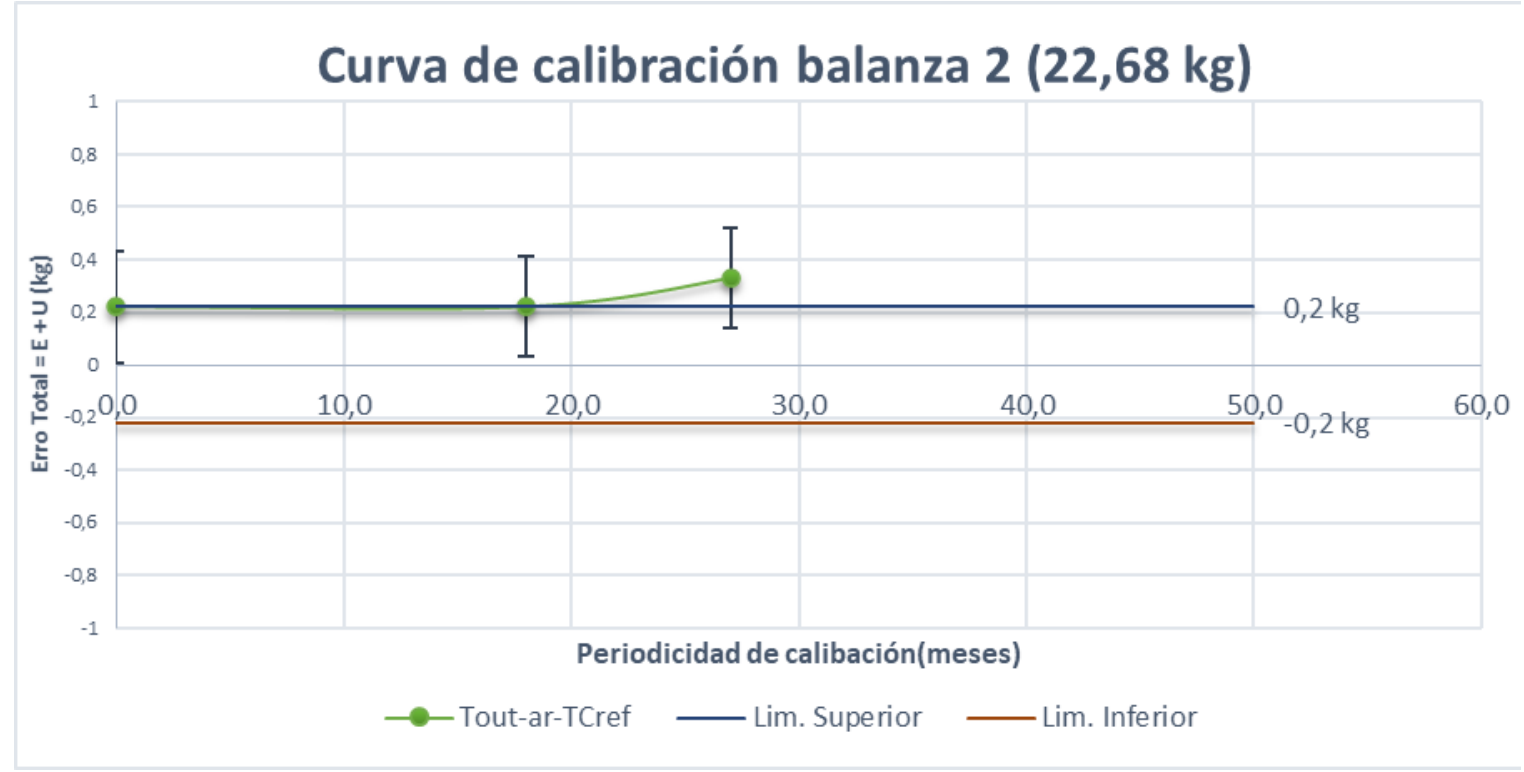

Fuente: Elaboración propia

De la Figura 2 podemos concluir que este instrumento tiende a comportarse de manera errónea ya que los errores están por fuera del rango de tolerancia permisible de este. Se recomienda disminuir el tiempo de calibración y realizarle mantenimiento con mayor frecuencia para poder ayudar al equipo a mantener un comportamiento funcional más estable.

Figura 3. Comportamiento de calibración de balanza 3

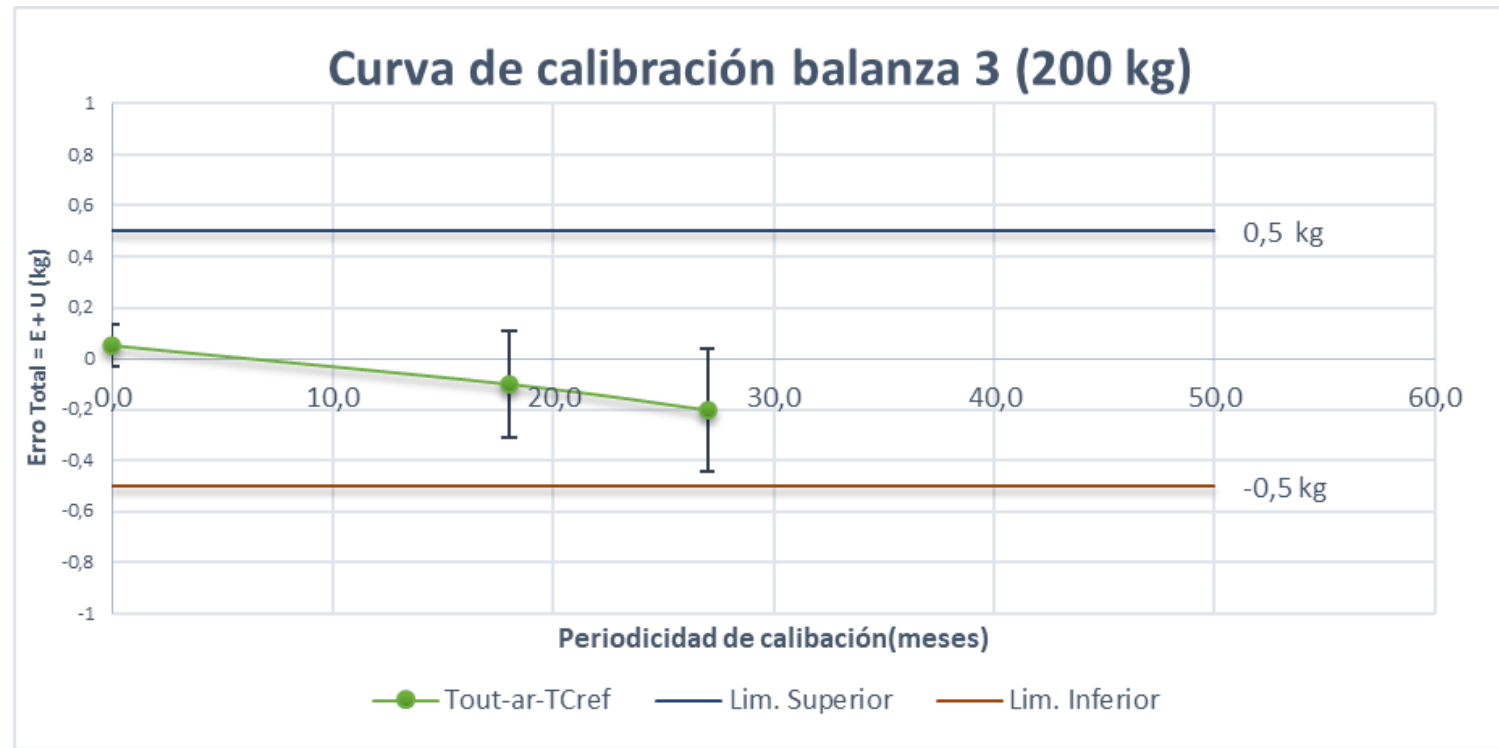

Fuente: Elaboración propia 
Analizando la Figura 3 podemos observar que a pesar que los errores se encuentren dentro del límite permitido, el instrumento tiende a aumentar su error a medida que pasa el tiempo por lo que es recomendable que se realicen planes de mantenimiento que ayuden a tener el instrumento en condiciones óptimas, ya que como se observa, si no se realizan correcciones éste llegará a presentar fallos más adelante y exista la posibilidad de que deba ser reemplazado.

\section{Aplicación de método de Schumacher}

Analizando los certificados de calibración se realizó una Tabla de confirmación metrológica que contiene la información presentada en la Tabla 6:

Tabla 6. Información de historial de certificados de calibración de balanzas seleccionadas

\begin{tabular}{|c|c|c|c|}
\hline Equipos & Calibración 2017 & Calibración 2018 & Calibración 2019 \\
\hline Balanza 1 $(140 \mathrm{~kg})$ & $\mathrm{F}$ & $\mathrm{C}$ & $\mathrm{C}$ \\
\hline Balanza 2 $22,6 \mathrm{~kg})$ & $\mathrm{F}$ & $\mathrm{F}$ & $\mathrm{F}$ \\
\hline Balanza 3 $200 \mathrm{~kg})$ & $\mathrm{C}$ & $\mathrm{C}$ & $\mathrm{C}$ \\
\hline
\end{tabular}

Fuente: Elaboración propia

Teniendo en cuenta los datos de la Tabla 6 y lo establecido por el método Schumacher, se tiene que la nueva periodicidad de calibración de las tres balanzas debe variar según lo siguiente:

Tabla 7. Resultado de método Schumacher para balanzas seleccionadas

\begin{tabular}{|c|c|c|}
\hline Balanza 1 (140 kg) & Balanza 2 (22,6 kg) & Balanza 3 (200 kg) \\
\hline$P$ & $M$ & $E$ \\
\hline
\end{tabular}

Fuente: Elaboración propia

- Balanza 1 (140 kg): Se debe mantener la misma periodicidad de calibración de 330 días mientras los resultados se mantengan iguales.

- Balanza 2 (22,6 kg): Debemos realizarle una máxima reducción de la periodicidad del 35\%, por lo que para esta balanza, que venía calibrándose cada 360 días, ahora pasará a calibrarse con una periodicidad de 234 días.

- Balanza 3 (200 kg): Dado el comportamiento de los errores que lleva esta balanza según los certificados de calibración, podemos hacer un aumento en la periodicidad del $20 \%$, por lo que su antigua periodicidad de calibración de 320 días pasará a ser de 256 días.

\section{Conclusiones}

En el presente trabajo se desarrolló una verificación del estado actual de los instrumentos no automáticos de pesaje de la Universidad del Atlántico que tuvo como alcance de proyecto el inventario de equipos no automáticos de pesaje de los laboratorios de nutrición y dietética ya que estos laboratorios tienen una variedad de balanzas considerable, donde se logró determinar que: 
José Daniel Hernández Vásquez, Norberto José Rodríguez-Medina, Albeiro Javier Fernández-Moreno,

Cristian Antonio Pedraza-Yepes

- Muchos de estos equipos están trabajando fuera de sus límites de tolerancia y errores máximos permisibles.

- Existen equipos que no cuentan con el adecuado mantenimiento de sus partes mecánicas y/o electrónicas.

- Hay equipos que están para calibración, debido a su uso y a que el tiempo entre la última calibración pasó la periodicidad sugerida para cada equipo.

Dadas estas observaciones, es necesario que el plan de mantenimiento sugerido en este proyecto siga llevándose a cabo teniendo como responsable de estas tareas al personal de mantenimiento y tutores de aulas pertinentes.

A manera de ejemplo se desarrolló la ejecución del plan de mantenimiento propuesto a cinco balanzas del inventario con el fin de establecer costos, verificar la ejecución de cada actividad propuesta y realizar la calibración de los equipos, tomando como objetos de estudio para ejecutar los métodos propuestos por la OIML D10 tres de estos, ya que eran los únicos que contaban con el historial necesario de calibraciones para llevarse a cabo el cálculo de periodicidad de calibración.

Con base a los resultados obtenidos de manera analítica y predictiva de las Tablas y Figuras de los métodos propuestos en este proyecto, podemos establecer que una combinación en el uso de estos dos sería la herramienta adecuada para garantizar las condiciones metrológicas de nuestros laboratorios, debido a su dificultad de aplicación media y confiabilidad alta.

Este documento servirá como guía base para un futuro plan de mantenimiento general que incluya cada uno de los equipos existentes en las instalaciones de la Universidad del Atlántico, así como también para que el algoritmo del Software diseñado y pueda ser agregado y ayude a complementar un Software con mayor información, ya sea mediante una interfaz de C++ o MathLab.

\section{Referencias bibliográficas}

1. HU, Jiawen; CHEN, Piao. Predictive maintenance of systems subject to hard failure based on proportional hazards model. Reliability Engineering \& System Safety, vol. 196, p. 106707. 2020.

2. Ahmed, A., Guozhu, J., Majeed, A., \& Salam, A. Uncertainty Measurement and Analysis for Quality and Reliability in Manufacturing Process. In IOP Conference Series: Materials Science and Engineering (Vol. 576, No. 1, p. 012007). IOP Publishing. July, 2019.

3. Ehrlich, C. Terminological aspects of the Guide to the Expression of Uncertainty in Measurement (GUM). Metrologia, 51(4), S145. 2014.

4. Universidad del Atlántico. Acreditación de programas. [En línea]. Disponible en:https://www. uniatlantico.edu.co/uatlantico/docencia/acreditacion/programas-acreditados. Septiembre, 2019.

5. Instituto colombiano de normas técnicas y certificación. Sistemas de gestión de calidad. Requisitos. NTC-ISO 9001. Bogotá D.C.: El Instituto. 2015. 33 p.

6. International Organization of Legal Metrology D-10, Guidelines for the determination of calibration intervals of measuring instruments. Edition 2007.

7. D. Medrano, \& B. Vega. Diseño e implementación de un plan integral de mantenimiento y calibración de las maquinas del laboratorio de procesos de manufactura de la Escuela Politécnica del Ejército. Escuela Politécnica del Ejército. Sede Sangolqui, Ecuador. 2012.

Revista Investigación e Innovación en Ingenierías, vol. 9, nº. 2, pp. 192-207, julio-diciembre 2021. DOI: https://doi.org/10.17081/invinno.9.2.4517 
8. E. Dumaguala. Gestión e implementación del plan de mantenimiento en los laboratorios del área de ingeniería mecánica en la Universidad Politécnica Salesiana sede Cuenca. Universidad Politécnica Salesiana sede Cuenca. Cuenca, Ecuador. 2014.

9. N. Montealegre. Diseño de un plan de mantenimiento preventivo para los equipos de los laboratorios pertenecientes a la facultad de ingeniería mecánica de la Universidad Tecnológica de Pereira. Universidad Tecnológica de Pereira. Pereira, Colombia. 2018.

10. E. Cruz, V. Branda. "Management of calibration intervals for temperature and static pressure transmitters applied to the natural gas industry", ELSEVIER. Vol. 24 pp.178-184. Mayo 2015.

11. J. Wang, Q. Zhang, W. Jiang. "Optimization of calibration intervals for automatic test equipment", ELSEVIER. Vol. 103 pp. 87-92. Junio 2017.

12. Vásquez, J. D. H., de Faro Orlando, A., Frota, M. N., \& de Oliveira, E. C. An alternative gravimetric measurement standard for calibration of liquid flow meters. Flow Measurement and Instrumentation, 58, 87-96. 2017.

13. Especialistas en Metrologías SAS. Metrología en Colombia. Colombia. [En línea]. Disponible en: https://www.especialistasenmetrologia.com/metrologia-en-colombia.html. Noviembre, 2018.

14. MONTGOMERY, Douglas C.; GARCíA, Rodolfo Piña. “Control estadístico de la calidad”. Limusa Wiley, 2004. Librovirtual.Disponibleen:http://dspace.ucbscz.edu.bo/dspace/bitstream/123456789/24701/1/13345. pdf

15. Hérnandez, E. Jiménez. “Diseño e implementación del sistema de aseguramiento metrológico en los laboratorios de la Universidad del Atlántico y creación del programa de mantenimiento preventivo a los equipos de medición". Tesis de pregrado. Universidad del Atlántico, Puerto Colombia. 2011.

16. Health o meter ${ }^{\circledR}$. Products Health o meter ${ }^{\circledR}$. [En línea]. Disponible en: https://www.homscales.com/ products

17. Mettler Toledo (2019, sept). Industrial weighing solutions. [En línea]. Disponible en: https://www. mt.com/int/es/home/products/Industrial_Weighing_Solutions/balances-scales. Septiembre, 2019.

18. Bernalo ${ }^{\circledR}$. Balanzas. [En línea]. Disponible en: https://www.bernalo.com.co/product-category/ balanzas/ Septiembre, 2019.

19. Detecto Industrias. Productos. [En línea]. Disponible en: http://www.detecto.co/basculas.html Septiembre, 2019.

20. KERN. Balanzas de precisión. [En línea] Disponible en: https://www.kern-sohn.com/shop/es/ balanzas-de-laboratorio/balanzas-de-precision/

21. BDco. (2019, sept). Lista de productos. [En línea]. Disponible en: https://www.balanzasdigitales.com/ brand/5-proscale Septiembre, 2019.

22. Baxtran. Basculas. [En línea]. Disponible en: https://www.baxtran.com/es/basculas.html. Septiembre, 2019.

23. [23] Científica SENNA. Balanzas granatarias. [En línea] Disponible en: https://cientificasenna.com/ producto/balanza-granataria-de-tres-brazos/. Abril, 2019.

24. Renovetec. Elaboración de planes de Mantenimiento. [En línea]. Disponible en: http://www.renovetec. com/931-guia-2-elaboracion-de-planes-de-mantenimiento. Agosto, 2019.

25. Instituto colombiano de normas técnicas y certificación. Instrumentos de pesaje de funcionamiento no automáticos. Requisitos metrológicos y técnicos. NTC 2031. Segunda edición. Bogotá D.C. 2014.

26. Jeannette Cristina Forero H. "Estrategias para definir el plan de confirmación metrológica". Instituto nacional de salud, Bogotá D.C. 2016. 